\title{
Tumescent Local Anesthesia for Hand Surgery: Improved Results, Cost Effectiveness, and Wide- Awake Patient Satisfaction
}

\author{
Donald Lalonde, Alison Martin \\ Department of Surgery, Dalhousie University, Saint John, NB, Canada
}

This is a review article of the wide-awake approach to hand surgery. More than 95\% of all hand surgery can now be performed without a tourniquet. Epinephrine is injected with lidocaine for hemostasis and anesthesia instead of a tourniquet and sedation. This is sedationfree surgery, much like a visit to a dental office. The myth of danger of using epinephrine in the finger is reviewed. The wide awake technique is greatly improving results in tendon repair, tenolysis, and tendon transfer. Here, we will explain its advantages

Keywords Epinephrine / Local anesthesia / Tourniquet

\author{
Correspondence: Donald Lalonde \\ Department of Surgery, Dalhousie \\ University, Hilyard Place, Suite C204, \\ 600 Main Street, Saint John, \\ NB E2K 1J5, Canada \\ Tel: +1-506-648-7950 \\ Fax: +1-506-652-8042 \\ E-mail: drdonlalonde@nb.aibn.com
}

No potential conflict of interest relevant to this article was reported.

Received: 5 Aug 2013 • Revised: 8 Sep 2013 • Accepted: 8 Sep 2013

pISSN: 2234-6163 • elSSN: 2234-6171 • http://dx.doi.org/10.5999/aps.2014.41.4.312 • Arch Plast Surg 2014;41:312-316

\section{PURPOSE OF THIS REVIEW PAPER}

This expanded review is intended to update the reader of new developments in Wide-Awake Local Anesthesia No Tourniquet (WALANT) hand surgery since only older and more limited review articles on the topic have been published thus far $[1,2]$

\section{EPINEPHRINE HEMOSTASIS IS SAFE IN THE FINGER, AND THE TOURNIQUET IS NO LONGER NECESSARY FORMOST TYPES OF HAND SURGERY}

The myth that epinephrine is not safe originated before 1950 . Procaine was the first and only synthetic injectable local anesthetic until 1948 when lidocaine was invented [3]. Procaine started with an acidic $\mathrm{pH}$ of 3.6 and became more acidic as it aged [4]. Old yellowish procaine was injected into patients in the days before 1972 when expiry dates were mandated in the USA [5]. In 1948, an alert by the Food and Drug Administration [6] in the USA warned that they had found procaine batches with a pH of 1 being injected into patients. Procaine necrosed fingers at this very acidic $\mathrm{pH}$, and epinephrine took the blame. Almost all 48 cases of finger loss caused by local anesthesia occurred before 1950. Most of the cases were with procaine without epinephrine, while fewer occurred when procaine was used with epinephrine [3]. There is still not one case of finger loss due to lidocaine with adrenaline reported anywhere in the world literature [7].

Two large studies with a total of more than 4,000 cases of epinephrine in the finger and hand have shown that phentolamine rescue is almost never necessary $[3,8]$. Thomson summarized all of the rest of the evidence that the use of epinephrine in the finger is safe [3]. If there is ever any concern about finger safety, $1 \mathrm{mg}$ of the alpha blocker phentolamine in 1 to $10 \mathrm{~mL}$ of saline can be injected wherever epinephrine has been injected in order 
to reverse the vasoconstriction [8]. The senior author has used epinephrine in the finger in more than 2,000 cases since the year 2000. He has not lost one finger, and he has never had to use phentolamine to reverse adrenaline vasoconstriction. Even a high dose of epinephrine $(1: 1,000)$ does not kill fingers [9].

Epinephrine hemostasis has removed the need for the tourniquet in most types of hand surgery. This has had a radical effect on the way much of the hand surgery is now performed worldwide. Most carpal tunnel surgery in Canada is now performed in minor procedure rooms outside of the main operating room with no sedation and no monitoring, and with field sterility (just 4 towels, a mask, and gloves only for the surgeon) [10]. Field sterility has been shown to have very low infection rates in the case of carpal tunnel surgery, even in the absence of preoperative antibiotics [11].

Visibility with epinephrine hemostasis is perfectly adequate, particularly if the patient is injected outside the operating room to give the epinephrine time to vasoconstrict [12]. The concern about visibility without a tourniquet is frequently brought up by surgeons who have never used the technique, but not by those who have experience with Wide-Awake Local Anesthesia No Tourniquet (WALANT).

\section{COST EFFECTIVENESS OF WIDE- AWAKE HAND SURGERY AND PATIENT SATISFACTION}

Removing the need for the tourniquet means that sedation is no longer required for most types of hand surgery [1]. The experience for most patients is very satisfying as it makes simple hand surgery like carpal tunnel release and trigger finger much like going to the dentist [13]. They just get up and go home after the surgery without having to recover from drugs. They no longer have to waste their time and money on unnecessary preoperative testing that was required for sedation, which is no longer needed now that tourniquet use and thus the related pain are eliminated [14]. Intraoperative monitoring is not required as the only two drugs that are used are lidocaine and epinephrine. These two drugs have been injected without monitoring in dental offices around the world for more than 60 years now with very few adverse reactions [15]. In addition, in most hospitals of the world, minor medical and surgical procedures with pure lidocaine with epinephrine have also been carried out without monitoring or intravenous insertion and with excellent safety records for many years. Eliminating all sedation eliminates all risks of sedation.

In addition, newer techniques in local anesthesia injection have almost completely eliminated the pain of the injections other than the discomfort of a 27-gauge needle in the wrist [16].

There are many cost savings associated with this approach as the anesthesiologist, the recovery room staff, and the preoperative testing costs are not required $[10,17,18]$. The senior author has routinely performed three carpal tunnel operations per hour with just one nurse at a leisurely pace for more than 15 years.

\section{TIPS TO MAIKE CARPAL TUNNEL AND TRIGGER FINGER SURGERY MORE EFFICIENT IN MINOR PROCEDURE ROOMS IN THE CLINIC OR OFFICE}

It has now been shown that the time to maximal vasoconstriction after injection of lidocaine and epinephrine is 25 minutes, not the previously believed 7 minutes [19]. We tell patients to bring a book to read as local anesthetic is like baking a cake; after you put it in the oven, you must wait at least half an hour for it to be ready. We inject the first three patients on stretchers outside the operating room and complete the paperwork before operating on the first one. After the first surgery, the nurse changes the room and brings in the second patient, while the surgeon injects the local anesthesia into the fourth patient. In this way, the surgeon wastes no time but does get to educate the patient during the injections and the surgery. The patients also like the opportunity to get their questions answered by the surgeon. We have found that this improved communication decreases our complication rate and increases our patient satisfaction rate. Carpal tunnel cases simply get a soft bandage, which has been shown to be superior to splinting [12].

We inject $20 \mathrm{~mL}$ of $1 \%$ lidocaine with 1:100,000 epinephrine for carpal tunnel: $10 \mathrm{~mL}$ in the wrist between the median and ulnar nerves, and $10 \mathrm{~mL}$ in the palm. This is considerably more than required, but we also almost never need to add more local anesthetic because of patient discomfort [20]. Lower concentrations of $1 / 4 \%$ lidocaine with 1:400,000 have also been effective in the hands we have treated if there are concerns regarding heart health. This technique can be easily shown to medical students who can then rapidly perform the task with very little pain. For a video on the technique, see the reference [21].

\section{TIPS TO DECREASE THE PAIN OF THE LOCAL ANESTHETIC INJECTIION}

A recent review [22] of how to inject local anesthesia so patients feel minimal pain during the injection process suggests the following: 1) buffer lidocaine and epinephrine $10: 1$ with $8.4 \%$ bi- 


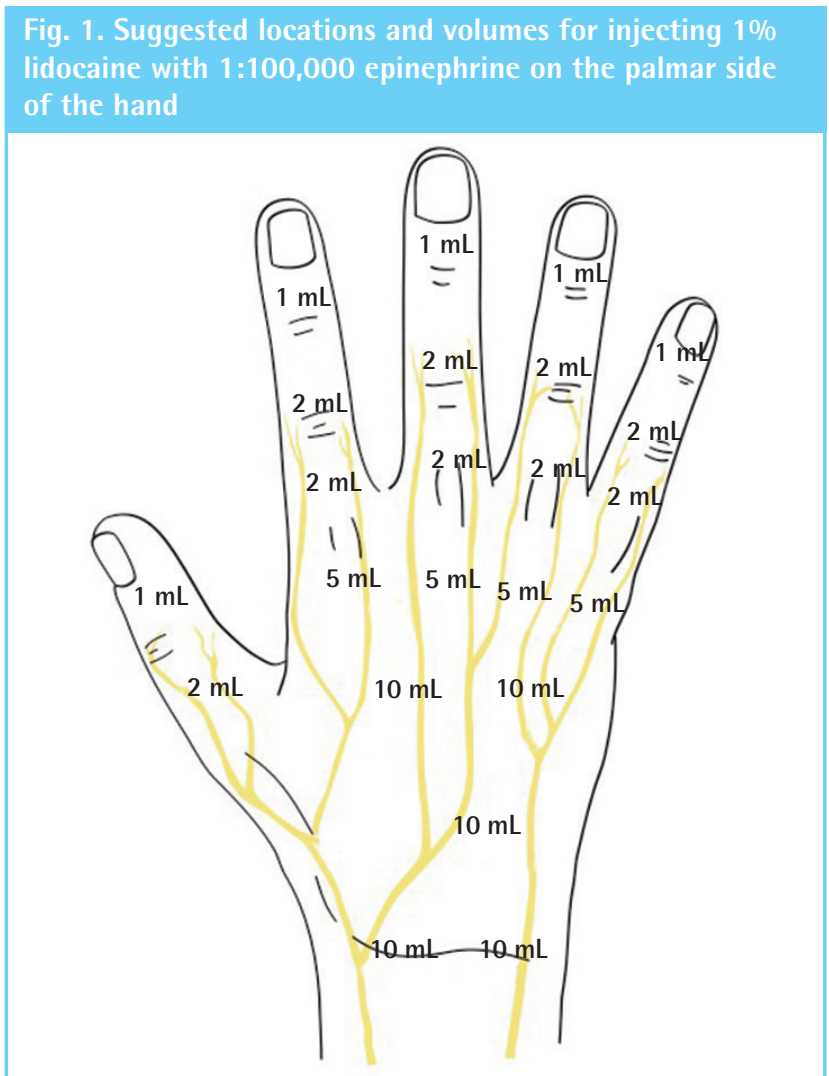

carbonate, 2) warm the local anesthetic solution to body temperature, 3 ) distract the patient to look away from the area of injection by using touch or pressure, 4) use a 27-gauge needle, 5) stabilize the syringe to avoid needle movement until the entry site is numb, 6) inject $0.5 \mathrm{~mL}$ of the local anesthetic perpendicularly subdermally and pause until the patient says that the needle pain is gone, 7) inject an additional $2 \mathrm{~mL}$ of the anesthetic before moving the needle, and then move antegrade very slowly with a $1 \mathrm{~cm}$ wheal of local anesthetic fluid always palpable or visible ahead of the needle ("blow slow before you go..."), 8) reinsert needles $1 \mathrm{~cm}$ inside the border of the blanched skin, 9) never put the needle into the nerve to elicit paresthesia, and 10) learn from the patients that you inject by asking them to tell you each time they experience pain during the injection process so that you know what you did wrong when they felt some pain after the introduction of the first needle. The usual mistake is to move very quickly, which lets the needle penetrate an area that has not yet been anesthetized.

In the case of a Zone 2 flexor tendon repair, inject $10 \mathrm{~mL}$ of local anesthetic in the palm without moving the needle in the most proximal area of the dissection in order to anesthetize the common digital nerves. Twenty minutes later, inject $2 \mathrm{~mL}$ of $1 \%$ lidocaine with 1:100,000 epinephrine in the middle of the volar subcutaneous fat (now painless because of the nerve blocks) in the proximal and middle phalanges. If you will be dissecting in
Fig. 2. Suggested locations and volumes for injecting 1\% lidocaine with 1:100,000 epinephrine on the dorsal side of the hand

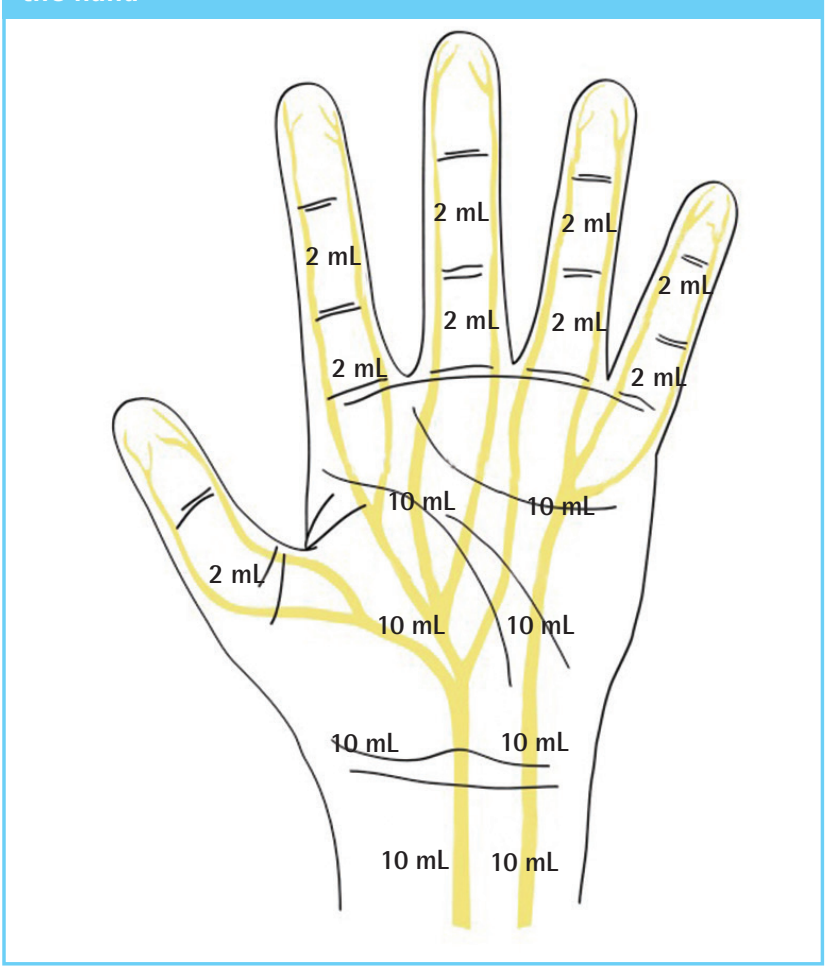

the distal phalanx, inject $1 \mathrm{~mL}$ of the above mix there. See Figs. 1,2 for suggested locations and volumes to inject $1 \%$ lidocaine with 1:100,000 epinephrine.

\section{IMPROVED RESULTS IN FLEXOR TENDON REPAIR SURGERY}

When patients are pain free and not sedated (no tourniquet), they can help the surgeon during the surgery. In the case of tenolysis, the patients can pull on the tendons and show the surgeon where they are stuck. The patients can also rupture the last bit of adhesion for the surgeon by actively pulling on the tendon. Finally, the patients also see and remember the active range of motion that they can achieve because they are not on drugs.

A cooperative comfortable patient can make a full fist and extend his fingers completely after a flexor tendon repair before the skin is closed. This does two things: 1) It shows the surgeon that the repair can fit through the pulleys, and hence, tenolysis will not be required later. If it does not fit through the pulleys, they are vented (partially divided) until the patient gets a full range of motion. This has decreased the rate of tenolysis [23].2) The ability to test the repair with full flexion and extension during the surgery also reveals a gap in some cases. Gaps occur because the repair was not tied sufficiently tightly. The gap would have led to rupture, but the surgeon sees it during the surgery 
and goes onto redo the repair so that there is no gap with full active flexion and extension before closing the skin. This has reduced rupture rates [24].

When a surgeon sees full fist flexion and full extension during the surgery without gapping, the therapist and the surgeon feel comfortable asking a patient to perform half a fist of flexion during the recovery phase with $45^{\circ}$ of true active metacarpal phalangeal joint (MP) extension from $90^{\circ}$ in the resting splint, $45^{\circ}$ of true active proximal interphalangeal joint (PIP) flexion, and $45^{\circ}$ of true active distal interphalangeal joint (DIP) flexion with active movement starting three days after the surgery [25]. We are now consistently obtaining better results because we are able to use a true active protected movement postoperatively.

\section{GETTING THE TENSION RIGHT IN TEND ON TRANSFER SURGERY}

We have all made tendon transfers too tight or too loose with patients asleep or sedated. When they are awake and cooperative, they can comfortably flex and extend transfers such as the extensor indicis to extensor pollicis longus before the skin is closed. If the transfer is too tight or too loose, it can be adjusted intraoperatively [26] in order to obtain a better result.

\section{PATIENTS HELP TO CHOOSE THE FINAL ANGLE IN JOINT FUSION}

When fusing thumb MP joints or finger IP joints, temporary $\mathrm{K}$ wires are placed across the joint. An awake patient takes the fingers and thumb through an entire range of movements to ensure that the fusion angles are ideal. If they are not, the $\mathrm{K}$ wires and angles can be adjusted before the skin is closed.

\section{CONCLUSIONS}

Hand surgery of a wide-awake patient is a safer, cheaper, and more enjoyable experience for the patient. It has improved intraoperative communication between the surgeon and the patient. It has also led to improvements in the results, particularly in the cases of tendon repair and tendon transfer.

\section{REFERENCES}

1. Lalonde DH. Reconstruction of the hand with wide awake surgery. Clin Plast Surg 2011;38:761-9.

2. Lalonde D, Martin A. Epinephrine in local anesthesia in finger and hand surgery: the case for wide-awake anesthesia. J Am Acad Orthop Surg 2013;21:443-7.
3. Thomson CJ, Lalonde DH, Denkler KA, et al. A critical look at the evidence for and against elective epinephrine use in the finger. Plast Reconstr Surg 2007;119:260-6.

4. Terp P. Hydrolysis of procaine in aqueous buffer solutions. Acta Pharmacologica et Toxicologica 1949;5:353-62.

5. Uri J, Adler P. The disintegration of procaine solutions. Curr Res Anesth Analg 1950;29:229-34.

6. Food and Drug Administration. Warning-procaine solution. JAMA 1948;138:599.

7. Denkler K. A comprehensive review of epinephrine in the finger: to do or not to do. Plast Reconstr Surg 2001;108: 114-24.

8. Nodwell T, Lalonde D. How long does it take phentolamine to reverse adrenaline-induced vasoconstriction in the finger and hand? A prospective, randomized, blinded study: the Dalhousie project experimental phase. Can J Plast Surg 2003;11:187-90.

9. Fitzcharles-Bowe C, Denkler K, Lalonde D. Finger injection with high-dose $(1: 1,000)$ epinephrine: Does it cause finger necrosis and should it be treated? Hand (N Y) 2007;2:5-11.

10. Leblanc MR, Lalonde J, Lalonde DH. A detailed cost and efficiency analysis of performing carpal tunnel surgery in the main operating room versus the ambulatory setting in Canada. Hand (N Y) 2007;2:173-8.

11. Leblanc MR, Lalonde $\mathrm{DH}$, Thoma $\mathrm{A}$, et al. Is main operating room sterility really necessary in carpal tunnel surgery? A multicenter prospective study of minor procedure room field sterility surgery. Hand (N Y) 2011;6:60-3.

12. Henry SL, Hubbard BA, Concannon MJ. Splinting after carpal tunnel release: current practice, scientific evidence, and trends. Plast Reconstr Surg 2008;122:1095-9.

13. Davison P, Cobb T, Lalonde D. The patient's perspective on carpal tunnel surgery related to the type of anesthesia: a prospective cohort study. Hand 2013;8:47-53.

14. Mustoe TA, Buck DW 2nd, Lalonde DH. The safe management of anesthesia, sedation, and pain in plastic surgery. Plast Reconstr Surg 2010;126:165e-76e.

15. Jeske AH. Xylocaine: 50 years of clinical service to dentistry. Tex Dent J 1998;115:9-13.

16. Strazar R, Lalonde D. Minimizing injection pain in local anesthesia. CMAJ 2012;184:2016.

17. Chatterjee A, McCarthy JE, Montagne SA, et al. A cost, profit, and efficiency analysis of performing carpal tunnel surgery in the operating room versus the clinic setting in the United States. Ann Plast Surg 2011;66:245-8.

18. Bismil M, Bismil Q Harding D, et al. Transition to total onestop wide-awake hand surgery service-audit: a retrospective review. JRSM Short Rep 2012;3:23. 
19. McKee DE, Lalonde DH, Thoma A, et al. Optimal time delay between epinephrine injection and incision to minimize bleeding. Plast Reconstr Surg 2013;131:811-4.

20. Lalonde DH. "Hole-in-one" local anesthesia for wide-awake carpal tunnel surgery. Plast Reconstr Surg 2010;126:1642-4.

21. Farhangkhoee H, Lalonde J, Lalonde DH. Teaching medical students and residents how to inject local anesthesia almost painlessly. Can J Plast Surg 2012;20:169-72.

22. Strazar AR, Leynes PG, Lalonde DH. Minimizing the pain of local anesthesia injection. Plast Reconstr Surg 2013;132: 675-84.

23. Lalonde DH. Wide-awake flexor tendon repair. Plast Re- constr Surg 2009;123:623-5.

24. Higgins A, Lalonde DH, Bell M, et al. Avoiding flexor tendon repair rupture with intraoperative total active movement examination. Plast Reconstr Surg 2010;126:941-5.

25. Lalonde D. How the wide awake approach is changing hand surgery and hand therapy: inaugural AAHS sponsored lecture at the ASHT meeting, San Diego, 2012. J Hand Ther 2013;26:175-8.

26. Bezuhly M, Sparkes GL, Higgins A, et al. Immediate thumb extension following extensor indicis proprius-to-extensor pollicis longus tendon transfer using the wide-awake approach. Plast Reconstr Surg 2007;119:1507-12. 